Revue européenne des sciences sociales

European Journal of Social Sciences

XLIV-134 | 2006

Quel(s) défi(s) pour les sciences sociales à l'heure de la mondialisation?

\title{
Publications scientifiques de Gérald Berthoud.
} 1962-2006

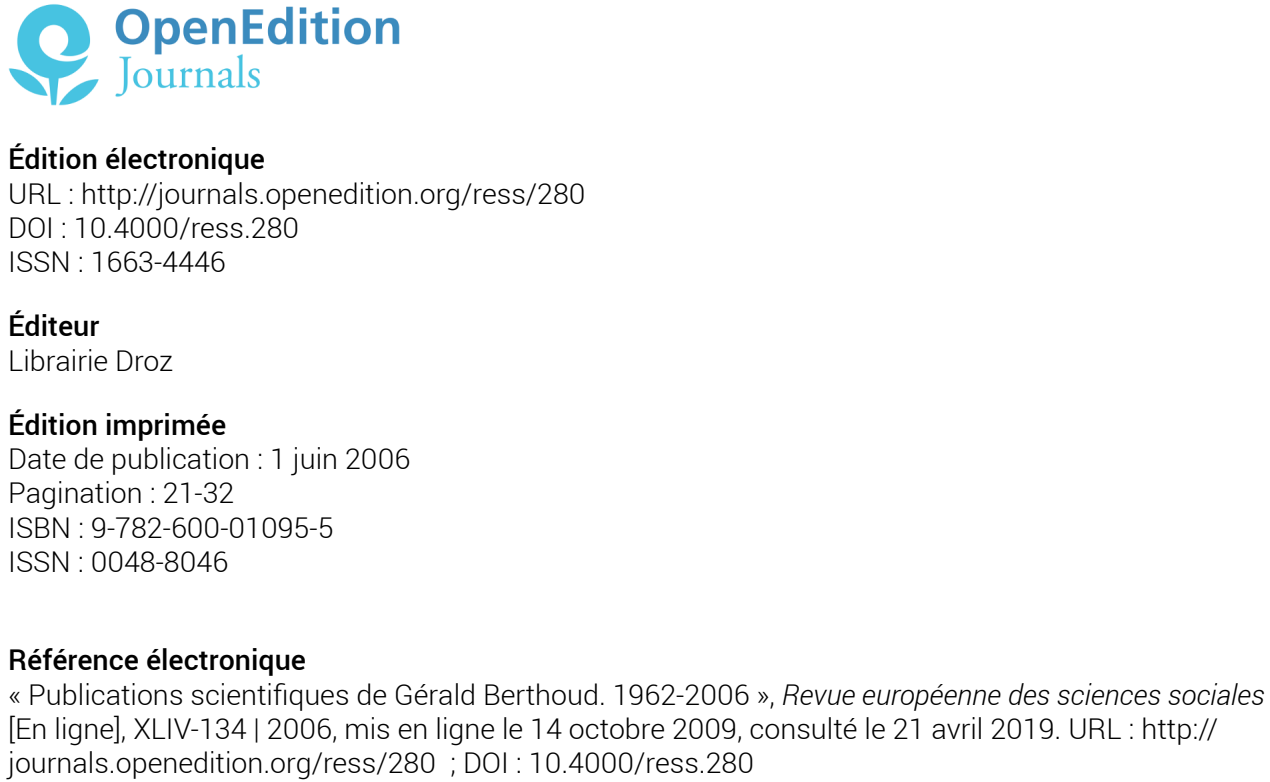

Édition électronique

URL : http://journals.openedition.org/ress/280

DOI : $10.4000 /$ ress. 280

ISSN : 1663-4446

Éditeur

Librairie Droz

Édition imprimée

Date de publication : 1 juin 2006

Pagination : 21-32

ISBN : 9-782-600-01095-5

ISSN : 0048-8046

\section{Référence électronique}

«Publications scientifiques de Gérald Berthoud. 1962-2006 », Revue européenne des sciences sociales [En ligne], XLIV-134 | 2006, mis en ligne le 14 octobre 2009, consulté le 21 avril 2019. URL : http:// journals.openedition.org/ress/280; DOI : 10.4000/ress.280 


\title{
PUBLICATIONS SCIENTIFIQUES DE GÉRALD BERTHOUD 1962-2006
}

\begin{abstract}
1962
«Figurines funéraires africaines». Musées de Genève (mars), 4 p.
\end{abstract}

1963

«Le culte des jumeaux chez les Fon du Dahomey ». Musées de Genève (avril), 4 p. «Magie, religion et art africains». Musées de Genève, 23 p.

\section{4}

«Le problème des couteaux de jet africains ». Musées de Genève (janvier), 4 p. «L'art de la Nouvelle Guinée». Musées de Genève (mars), 4 p.

«La société religieuse 'Die' chez les Gouro de côte d'Ivoire». Musées de Genève (octobre), 4 p.

«Considérations sur la culture matérielle des Kuba». Bulletin annиel du Musée d'ethnographie de Genève 7: 49-60.

\section{5}

«Réflexions sur l'art africain». Musées de Genève (février), 4 p.

«Le terrain, laboratoire de l'ethnologue». Musées de Genève (septembre), 4 p. «Essai historique sur les Ganawuri du Plateau (Nord du Nigeria)». Bulletin annuel du Musée d' ethnographie de Genève 8: 15-38.

\section{6}

«'Nzom', une cithare du Plateau (Nord du Nigeria)». Musées de Genève (février), $4 \mathrm{p}$.

«Une tribu du Nigeria». Publications du Musée d'ethnographie de Genève, 12 p.

\section{7}

«Pêcheurs ganawuri (Nigeria)». Musées de Genève (septembre), 4 p.

Changements économiques et sociaux de la montagne. Vernamiège en Valais. Préface d'André Leroi-Gourhan. Berne: Francke (publications de la Société suisse des sciences humaines, $\left.n^{\circ} 8\right), 237 \mathrm{p}$. 


\section{9}

«Les Ganawuri du Nigeria: facteurs techniques, économiques et religieux de la production matérielle». Bulletin annuel du Musée d' ethnographie de Genève 12: 9-91.

\section{0}

«La validité des concepts de 'multicentricité'» et de 'sphères d'échange' en anthropologie économique». Archives suisses d' anthropologie générale 34: 35-64.

«Anthropologie sociale et parenté. Quelques réflexions critiques sur 'Kinship and the social order' de Fortes». Archives suisses d'anthropologie générale 34: $140-146$.

«Introduction: Dynamics of ownership in the Circum Alpine Area ». Anthropological Quarterly (Washington D.C.) 45: 117-124.

«From peasantry to capitalism: the meaning of ownership in the Swiss Alps ». Anthropological Quarterly (Washington D.C.) 45 : 177-195.

«L'évolution politique de l'Afrique occidentale». Dans L'Afrique occidentale: développement et société. Publication du Centre international de criminologie comparée (Université de Montréal), p. 67-87.

«Psychologie économique africaine: éléments d'une recherche interdisciplinaire par J. Binet». American Anthropologist 74: 1402.

1973

«Structure et genèse d'une communauté alpine suisse». Dans L'homme, hier et aujourd' hui. Recueil d'études en hommage à André Leroi-Gourhan. Paris: Cujas, p. 643-653.

«Comment on 'Anthropology and Colonialism' by D. K. Lewis». Current Anthropology 14: 591-592.

«Comment on 'Two blades of grass: rural cooperatives in agricultural modernization’ by P. Worsley (ed.)». American Anthropologist 75: 997-998.

«Compte rendu de 'Ethnologie régionale 1: Afrique - Océanie' par J. Poirier (éd.)». Revue canadienne des études africaines 7: 351-355.

1974

«Compte rendu de 'Introduction à deux théories d'anthropologie sociale' par L. Dumont». American Anthropologist 76: 99-100.

«Développement et formation communautaire: les Ganawuri du Nigeria». Anthropologica (Ottawa) XVI, 1: 75-96.

«La signification sociale des 'biens de prestige' dans les formations lignagères africaines ». Revue canadienne des études africaines / Canadian Journal of African Studies VIII, 2: 307-324. 


\section{6}

«L'ambivalence de la production. Logique communautaire et logique capitaliste» (en coll. avec F. Sabelli). Cahiers de l' IUED (Genève) 3, 188 p.

«L'identité et l'altérité. Pour une confrontation de l'épistémologie génétique et de l'anthropologie critique». Revue européenne des sciences sociales 38-39: 471-494.

\section{7}

«Plaidoyer pour l'autre». Revue européenne des sciences sociales 41: 233-249. «Lecture anthropologique des Alpes. L'exemple du Valais». Bulletin d'études préhistoriques alpines 8-9: 53-66.

«La première société autogestionnaire». Autogestion (Paris) 39: 23-50.

«Anthropologie de la marginalité: Bagnes au XIX ${ }^{e}$ siècle». Alliance culturelle romande $23: 80-86$.

«Genetic epistemology, marxism, and anthropology». Dans The concept and dynamics of culture, B. Bernardi (ed.), The Hague, Paris: Mouton, p. 295-311.

\section{8}

«Etat-nation et ethno-résistance». Pluriel 15: 47-59.

«Une pensée contre l'Etat. Hommage à Pierre Clastres ». Autogestion (Paris) 40: 101-117.

\section{9}

«Gros hommes ganawuri et chefferies Rukuba. Deux modes communautaires de consumation de la richesse». Anthropologie et Sociétés (Québec) 3, 1:129-146.

«Our Obsolete Production Mentality: the Heresy of the Communal Formation» (en coll. avec F. Sabelli). Current Anthropology 20, 4: 745-760.

«Autogestion et souveraineté communautaire: l'éclairage de l'anthropologie critique ». Autogestion (Paris) 43: 7-23.

\section{0}

«Pièges productivistes et défis technologiques ». Autogestion (Paris) 4 (nouvelle série): 459-472.

«Le piège des besoins». Cahiers de l’ IUED (Genève) 11: 157-178.

«Comment on 'Challenging Anthropology: a critical introduction to social and cultural anthropology' par D. H. Turner et G. A. Smith (eds)». Anthropologie et Sociétés (Québec) 4(1): 186-190.

\section{1}

«Anthropologie critique et critique de l'épistémologie génétique». Revue européenne des sciences sociales 54-55: 183-204 (version légèrement modifiée de l'article de 1976). 
«La propriété: entre la nature et la culture» (en coll. avec G. Busino). Revue européenne des sciences sociales 59: 17-54.

\section{2}

Plaidoyer pour l'Autre. Essais d'anthropologie critique. Genève: Droz, 284 p. «Adaptation et résistance. Essai de relecture des cultes du cargo » (en coll. avec

M. Kilani). Cahiers Internationaux de Sociologie (Paris) LXXIII: 267-292. «Altérité et développement». Bulletin du M.A.U.S.S. (Paris) 1: 15-24.

«Le paradoxe communautaire: l'inégalité créatrice d'égalité». Bulletin $d u$ M.A.U.S.S. (Paris) 3-4: 96-103.

\section{3}

«Défense de la diversité ». Autogestions (Paris) 12-13: 175-180.

«La vie, un capital à gérer?». Bulletin du M.A.U.S.S. (Paris) 7: 67-80.

«Débat autour de 'Plaidoyer pour l'Autre'» (en coll. avec F. Alberoni, G. Balandier, G. Busino, M. Maffesoli et F. Sabelli). Revue européenne des sciences sociales 65 : $241-276$.

\section{4}

«Between Tradition and Modernity: the Example of Part Time Agriculture in Mountain Areas » (en coll. avec M. Kilani). Mezzogiorno d'Europa (Naples) 3: 369-408.

«Fra tradizione e modernità: l'esempio dell'agricoltura a tempo parziale in zona di montagna» (en coll. avec M. Kilani). Mezzogiorno d'Europa (Naples) 3: 391-430.

«Enseignement et recherche en anthropologie culturelle et sociale. L'exemple de l'agriculture en milieu alpin» (en coll. avec M. Kilani). Uni-Lausanne 38: 1-15. «L développement contre la vie». Revue Tiers Monde (Paris) 100: 871-880.

«La nébuleuse du don: essai de clarification». Bulletin du M.A.U.S.S. (Paris) 9: 139-142.

«La subordination féminine: affirmation discutable ou débat?». Bulletin du M.A.U.S.S. (Paris) 10: 11-21.

«Pour Louis Dumont» (en coll. avec G. Busino). Revue européenne des sciences sociales $68: 5-6$.

«De la comparaison» (en coll. avec G. Busino). Revue européenne des sciences sociales $68: 169-192$.

\section{5}

Sciences sociales et défi de la sociobiologie (éd.). Actes du I ${ }^{\text {er }}$ colloque annuel organisé par le Groupe d'Etude «Pratiques Sociales et Théories ». Revue européenne des sciences sociales 69, $376 \mathrm{p}$.

«Sciences sociales et biologie: un dialogue difficile». Revue européenne des sciences sociales 69: 5-19. 
«L'égoïsme, c'est la vie !». Revue européenne des sciences sociales 69: 255-278. «Entre l'anthropologue et le missionnaire. La contribution d'Henri-Alexandre Junod (1863-1934)». Dans Le visage multiplié du monde. Quatre siècles d'ethnographie à Genève. Musée d'ethnographie de Genève, p. 59-74 (version remaniée dans Revue européenne des sciences sociales 71, 1985).

«L'arche de Noé». Dans La forteresse européenne et les réfugiés. Lausanne: Editions d'en bas (édité par la Ligue suisse des droits de l'homme), p. 191-195.

«Periphery of the centre: economic vestige or cultural resistance? Mountain agriculture in Valais». Dans Endogenous development: a myth or a path? Problems of economic self-reliance in the european periphery, S. Musso (ed.), Tilburg: EADI, p. 175-196.

«L'enjeu de la tradition et de la modernité: l'agriculture de montagne en milieu alpin » (en coll. avec M. Kilani). Cahiers de la Société suisse d' histoire économique et sociale $4: 33-44$.

La démarche comparative. L'exemple de Karl Polanyi. Université de Lausanne, Institut d'anthropologie et de sociologie (collection «Cours, séminaires et travaux $\left.\gg n^{\circ} 5\right), 74 \mathrm{p}$.

\section{6}

La comparaison en sciences humaines et sociales (éd. en coll. avec G. Busino). Actes du $\mathrm{II}^{\mathrm{e}}$ colloque annuel organisé par le Groupe d'Etude «Pratiques Sociales et Théories ». Revue européenne des sciences sociales 72, $216 \mathrm{p}$.

«La comparaison: une idée ambiguë». Revue européenne des sciences sociales 72: 5-15.

«L'ambivalenza della produzione. Logiche comunitarie e logica capitalista (en coll. avec F. Sabelli). Napoli : Liguori, 149 p. (traduction de «L'ambivalence de la production», 1976).

«Autour de la race d'Hérens. Passions et enjeux (en coll. avec M. Kilani). Dans Le pays où les vaches sont reines, Y. Preiswerk et B. Crettaz (éds), Sierre: Monographic, p. 465-490.

«Le développement: entre l'impasse et le mirage». Dans Il était une fois le développement, G. Rist et F. Sabelli (éds), Lausanne: Editions d'en bas, p. 123-133.

«L'appât du développement». Bulletin du M.A.U.S.S. (Paris) 17: 103-112.

«L'économie en question: la position de Karl Polanyi». Bulletin du M.A.U.S.S. (Paris) 18: 53-104.

«Dichotomie ou totalité? Les vues de Polanyi sur l'homme et la société ». Bulletin du M.A.U.S.S. (Paris) 19: 115-137.

«La relativité pervertie». Bulletin du M.A.U.S.S. (Paris) 20: 55-70.

Vers une anthropologie générale. L'économique en perspective. Université de Lausanne, Institut d'anthropologie et de sociologie (collection «Cours, séminaires et travaux $\left.\gg \mathrm{n}^{\circ} 7\right), 154 \mathrm{p}$. 


\section{7}

«La connaissance anthropologique: un éclairage de la modernité ». Dans L'université en question (actes du colloque du $450^{\circ}$ anniversaire de la Fondation de l'Académie de Lausanne). Publications de l'Université de Lausanne LXX, p. 79-88.

Raison et relativité des valeurs (éd. en coll. avec G. Busino). Actes du $\mathrm{III}^{\mathrm{e}}$ colloque annuel organisé par le Groupe d'Etude «Pratiques Sociales et Théories ». Revue européenne des sciences sociales 74, 233 p.

«La raison de l'autre ou l'autre de la raison?». Revue européenne des sciences sociales $74: 135-160$.

«Le piège des idées reçues». Bulletin du M.A.U.S.S. (Paris) 22: 193-204.

«De la société à la sociétique?». Uni-Lausanne 53: 41-42.

«La modernité: vérité culturelle?». Bulletin du M.A.U.S.S. (Paris) 24: 33-51.

\section{8}

De l'utilité de la connaissance? (éd. en coll. avec G. Busino). Actes du IV colloque annuel organisé par le Groupe d'Etude «Pratiques Sociales et Théories ». Revue européenne des sciences sociales 79, 225 p.

«De l'utilité de la connaissance?» (en coll. avec G. Busino). Revue européenne des sciences sociales 79: 201-224.

L'économie dans la société (éd. en coll. avec G. Busino). Actes du V $\mathrm{V}^{\mathrm{e}}$ colloque annuel organisé par le Groupe d'Etude «Pratiques Sociales et Théories». Revue européenne des sciences sociales 82, $264 \mathrm{p}$.

«L'économie dans la société». Revue européenne des sciences sociales 82: 5-18.

«Le corps humain comme marchandise. Valeurs universelles et vérité du marché». Revue européenne des science sociales 82: 247-264.

«La passion de l'élevage en Valais » (en coll. avec M. Kilani). Uni-Lausanne 56: 12-15.

«Pensée religieuse: survivance ou permanence? Un éclairage anthropologique». Revue du M.A.U.S.S. (Paris) 1: 154-172.

\section{9}

«Droits de l'homme et savoirs anthropologiques». Dans Identité: évolution ou différence? L. K. Sosoe (éd.), Fribourg: Editions universitaires, p. 137-166.

«Pensée religieuse: survivance ou permanence? Un éclairage anthropologique» (reprise du texte de 1988). Dans L'Islam: une religion, P. Gisel et J. Waardenburg (éds.), Genève: Labor et Fides, p. 53-72.

«Le principe d'utilité et les 'restes'. De la division des tâches entre l'économie politique et la sociologie». Revue du M.A.U.S.S. (Paris) 6: 97-116.

«Castoriadis et la critique des sciences sociales ». Revue européenne des sciences sociales $86: 441-456$. 
«Entwicklungspolitik zwischen Sackgasse und Hirngespinst». Dans Das Märchen von der Entwicklung, G. Rist et F. Sabelli (Hrsg.), Zürich: Rotpunktverlag, p. 137-147 (traduction de «Il était une fois le développement», 1986).

«Le corps humain comme marchandise» (version abrégée du texte de 1988). Revue du M.A.U.S.S. (Paris) 3: 96-113.

\section{0}

Les intellectuels: déclin ou essor? (éd. en coll. avec G. Busino). Actes du VI colloque annuel organisé par le Groupe d'Etude «Pratiques Sociales et Théories ». Revue européenne des sciences sociales 87, 280 p.

«Les intellectuels: déclin ou essor?» (en coll. avec G. Busino). Revue européenne des sciences sociales $87: 251-278$.

«Identité régionale et passion de l'élevage en Valais: une approche anthropologique» (en coll. avec M. Kilani). Ethnologica Helvetica 13-14: 109-113.

«Toward a comparative approach: the contribution of Karl Polanyi». Dans The life and work of Karl Polanyi, K. Polanyi-Levitt (ed.), Montreal: Black Rose, p. 171-182.

«Modernity and Development». The European Journal of Development Research 2(1): 22-35.

«Le métissage de la pensée». Cahiers de l'IUED (Genève) 19: 17-43.

\section{1}

«Races de bétail, modes d'élevage et identités régionales. La vache: corps, symbole et décor» (en coll. avec B. Crettaz et Y. Preiswerk). Schweizerischen Archiv für Volkskunde 87: 189-208.

«The human body as a commodity». Dans The Legacy of Karl Polanyi. Market, State and Society at the end of the Twentieth Century, M. Mendell and D. Salée (eds), New York: St. Martin's Press, p. 109-118.

«La vache: corps, symbole et décor» (en coll. avec B. Crettaz et Y. Preiswerk). Dans Vache d' utopie, Genève: Slatkine (Itinéraires Amoudruz VII), p. 13-158. «Le marché comme simulacre du don?». Revue du M.A.U.S.S. (Paris) 11: 72-89. «Le marché comme simulacre du don?» (suite). Revue du M.A.U.S.S. (Paris) 12: 79-96.

L'homme et la société. Dix ans après Piaget (éd. en coll. avec G. Busino). Actes du VII ${ }^{\mathrm{e}}$ colloque annuel organisé par le Groupe d'Etude «Pratiques Sociales et Théories ». Revue européenne des sciences sociales 89, 256 p.

«L'homme et la société. Dix ans après Piaget». Revue européenne des sciences sociales $89: 5-12$.

«Somlò et l'ordre généralisé du don». Revue du M.A.U.S.S. (Paris) 14: 83-92.

Les techniques: enjeux humains et sociaux (éd. en coll. avec G. Busino). Actes du VIII colloque annuel organisé par le Groupe d'Etude «Pratiques Sociales et Théories ». Revue européenne des sciences sociales 91, 216 p.

«Médiation technique et relations sociales ». Revue européenne des sciences sociales 91: 97-111. 
«L'extériorisation informatique». Dans Actes du colloque «L'ordinateur a 40 ans», B. Galland (éd.), Lausanne: Ecole polytechnique fédérale, p. 211-219.

\section{2}

Vers une anthropologie générale. Modernité et altérité. Genève: Droz, 294 p. Les formes de la violence (éd. en coll. avec G. Busino). Actes du IX colloque annuel organisé par le Groupe d'Etude «Pratiques Sociales et Théories». Revue européenne des sciences sociales 94, $184 \mathrm{p}$.

«De la violence primordiale». Revue européenne des sciences sociales 94: 29-41. «Market». Dans The Development Dictionary. A Guide to Knowledge as Power, W. Sachs (ed.), London: Zed Books, p. 70-87.

«Anthropologie générale ou clôture disciplinaire?». Revue du M.A.U.S.S. (Paris) 15-16: 201-214.

«'Une science générale de l'homme'. L'œuvre d'Alexandre-César Chavannes». Annales Benjamin Constant (Lausanne) 13: 29-41.

\section{3}

La démocratie: une et multiple (éd. en coll. avec G. Busino). Actes du $\mathrm{X}^{\mathrm{e}}$ colloque annuel organisé par le Groupe d'Etude «Pratiques Sociales et Théories». Revue européenne des sciences sociales $97,280 \mathrm{p}$.

«Vers une démocratie marchande?». Revue européenne des sciences sociales 97 : 243-252.

«Esprit de sacrifice et secret du don». Revue semestrielle du M.A.U.S.S. (Paris) 1: 78-89.

«La société contre le don? Corps humain et technologies biomédicales ». Revue semestrielle du M.A.U.S.S. (Paris) 1: 257-274.

«Markt». Dans Wie im Westen so auf Erden, W. Sachs (Hrsg.), Hamburg: Rowohlt, p. 218-247 (traduction de «Market», 1992).

«Droits de l'homme et identité culturelle». Dans Universalisme-relativisme. Un débat d'actualité, G. Berthoud, P. Centlivres, C. Giordano et M. Kilani (éds), Fribourg: Presses universitaires, p. 99-122.

«Modernité et figures sacrificielles». Bulletin du Centre protestant d'études (Genève) 6: 19-25.

«Modernité et altérité. Un cheminement intellectuel». Revue semestrielle $d u$ M.A.U.S.S. (Paris) 2: 52-60.

\section{4}

«Il mercato come simulacro del dono?». Dans Il dono perduto e ritrovato, Roma: Manifestolibri, p. 47-67 (traduction partielle de «Le marché comme simulacre du don?», 1991).

«L'économie: un ordre généralisé? Les ambitions d'un prix Nobel». Revue semestrielle du M.A.U.S.S. (Paris) 3: 42-59. 
«Droits de l'homme et identité culturelle». Dans L'homme respecté, E. Fuchs et W. Ossipow (éds), Genève: Labor et Fides, p. 57-68 (reprise du texte de 1993). La théorie sociale aujourd' hui: bilan et perspectives (éd. en coll. avec G. Busino). Actes du XI colloque annuel organisé par le Groupe d'Etude «Pratiques Sociales et Théories ». Revue européenne des sciences sociales 99, 328 p.

\section{5}

«Que nous dit l'économie?». Dans L'économie dévoilée. Du budget familial aux contraintes planétaires. Paris: Editions Autrement (Série Mutations no 159), p. 61-73.

«Entre la règle de l'intérêt et la nécessité du sacrifice». Revue semestrielle $d u$ M.A.U.S.S. (Paris) 5: 101-117.

Pratiques sociales et théories. Les discordes des universitaires (en coll. avec G. Busino). Genève: Droz, 335 p.

\section{6}

«Economic Beliefs and Moral Responsibility». Dans The Responsible Scholar. Ethical Considerations in the Humanities and Social Sciences, G. Berthoud et B. Sitter-Liver (eds.), Canton, Massachusetts, USA: Watson, p. 133-149.

Mauss : hier et aujourd' hui (éd. en coll. avec G. Busino). Actes du XII colloque annuel organisé par le Groupe d'Etude «Pratiques Sociales et Théories». Revue européenne des sciences sociales 105, 284 p.

«Pourquoi Marcel Mauss?». Revue européenne des sciences sociales 105: 7-20. «Le don maussien: origine et fondement». Revue européenne des sciences sociales 105: 163-179.

«L'impératif marchand». Nouveaux Cahiers de l'IUED (Genève) 5: 175-184.

«L'unité-de-l'humanité-dans-la-diversité». Dans Genève métisse. De la Genève internationale à la cité pluriculturelle, L. Aubert, B. Crettaz, M. Graber et al. (éds), Genève: Zoé, p. 197-202.

\section{7}

«Entre bien-être et survie». Dans 50 Jahre. Auf dem Weg in die Zukunft, B. SitterLiver et C. Pfaff (Hrsg.), Bern: SAGW (publication à l'occasion du 50 anniversaire de l'Académie suisse des sciences humaines et sociales), p. 49-60.

«Préface». Dans Le rythme comme médiation. Mouvement Techno et nouvelles technologies, R. Meichtry, D. Rossé et J. Vacheron. Université de Lausanne, Institut d'anthropologie et de sociologie (collection «Pratiques et Théories des Sciences et des Techniques » $\left.\mathrm{n}^{\circ} \mathrm{II}\right), \mathrm{p} .7-8$.

Sciences et techniques dans la société (éd. en coll. avec G. Busino). Actes du XIII ${ }^{\mathrm{e}}$ colloque annuel organisé par le Groupe d'Etude «Pratiques Sociales et Théories». Revue européenne des sciences sociales 108, 312 p.

«Le spectre de la survie». Revue européenne des sciences sociales 108: 301-311. «La 'cage de fer' du capitalisme intégral». Revue semestrielle du M.A.U.S.S. (Paris) 9: 91-110. 
«'Recomposer le tout'. Le pluralisme méthodologique de Mauss ». Revue semestrielle du M.A.U.S.S. (Paris) 10: 221-240.

La machine universelle. Extraits de travaux d'étudiants (éd. en coll. avec D. Cerqui Ducret). Université de Lausanne, Institut d'anthropologie et de sociologie (collection «Pratiques et Théories des Sciences et des Techniques » $\mathrm{n}^{\circ}$ III), $146 \mathrm{p}$.

«The seductive market» (en coll. avec A. Rotstein). Dans The Milano Papers. Essays in Societal Alternatives, M. Cangiani (ed.), Montreal: Black Rose Books, p. 1-15.

«De l'anthropologie ou de l'unité-dans-la-diversité». Berne: Fonds national suisse de la recherche scientifique, Bulletin I Sciences humaines et sociales 19: 3-6.

\section{8}

Mémoire et savoirs à l'ère de l' information (éd. en coll. avec G. Busino). Actes du $\mathrm{XIV}^{\mathrm{e}}$ colloque annuel organisé par le Groupe d'Etude «Pratiques Sociales et Théories ». Revue européenne des sciences sociales 111, 222 p.

«Mémoire et savoirs à l'ère de l'information». Revue européenne des sciences sociales $111: 5-15$.

«Repenser le 'double mouvement'». Dans La modernité de Karl Polanyi. J.-M. Servet, J. Maucourant et A. Tiran (éds), Paris: L'Harmattan, p. 363-381.

«L'épreuve du potlatch. Entre intérêt, libéralité et prodigalité». Revue semestrielle du M.A.U.S.S. (Paris) 12: 294-310.

«Mercato». Dans Dizionario dello sviluppo, W. Sachs (ed.), Torino: Gruppo Abele, p. 85-113 (traduction de «Market», The Development Dictionary, 1992).

1999

«Des limites de l'homme normal». Revue européenne des sciences sociales 113 : 59-76.

«Un précurseur de Mauss. Felix Somlò et la question du don». Social Anthropology (Cambridge, UK) 7(2): 189-202.

Animalité et humanité. Autour d'Adolf Portmann (éd. en coll. avec G. Busino). Actes du $\mathrm{XV}^{\mathrm{e}}$ colloque annuel organisé par le Groupe d'Etude «Pratiques Sociales et Théories». Revue européenne des sciences sociales 115, 272 p. «Homo complexus». Revue européenne des sciences sociales 115: 185-198.

Dialogue avec Marcel Mauss. Université de Lausanne, Institut d'anthropologie et de sociologie (collection «Cours, séminaires et travaux» $\mathrm{n}^{\circ} 20$ ), $88 \mathrm{p}$.

\section{0}

Paroles reçues. Du bon usage des sciences sociales (en coll. avec G. Busino). Genève: Droz, 432 p.

La «société de l'information»: une idée confuse? (en coll. avec D. Cerqui, F. Clément, F. Ischy et O. Simioni). Université de Lausanne: Institut d'anthropologie et de sociologie (collection «Pratiques et Théories des Sciences et des Techniques » $\left.\mathrm{n}^{\circ} \mathrm{VI}\right), 141 \mathrm{p}$. 
Limites de l'éthique et action politique (éd.). Actes du XVI colloque annuel organisé par le Groupe d'Etude «Pratiques Sociales et Théories». Revue européenne des sciences sociales $118,184 \mathrm{p}$.

«Limites de l'éthique et action politique». Revue européenne des sciences sociales $118: 5-6$.

«La 'société de l'information': l'utopie du $\mathrm{XXI}^{\mathrm{e}}$ siècle?». Revue européenne des sciences sociales 118: 163-180.

«La générosité, une valeur de réserve». Les cahiers protestants (Lausanne) 6: 14-21.

\section{1}

«The 'spirit of the Alps' and the making of political and economic modernity in Switzerland». Social Anthropology (Cambridge, UK) 9(1): 81-94.

\section{2}

«Un monde surveillé». Dans Les grandes mutations, XXXVIII ${ }^{\text {es }}$ Rencontres Internationales de Genève 2001. Lausanne: L'Age d'Homme, p. 132-137.

La société de l'information: état des lieux (éd.). Actes du XVIII ${ }^{\mathrm{e}}$ colloque annuel organisé par le Groupe d'Etude «Pratiques Sociales et Théories ». Revue européenne des sciences sociales $123,226 \mathrm{p}$.

«Entre discours et pratiques: esquisse d'un état des lieux de la société de l'information» (en coll. avec D. Cerqui, F. Fassa et F. Ischy). Revue européenne des sciences sociales $123: 5-19$.

«L'horizon d'une surveillance omniprésente?». Revue européenne des sciences sociales 123 : $155-168$.

Histoire, philosophie et sociologie des sciences. Pour un état des lieux des rapports établis et des problèmes ouverts (éd. en coll. avec G. Busino). Actes du XIX ${ }^{\mathrm{e}}$ colloque annuel organisé par le Groupe d'Etude «Pratiques Sociales et Théories». Revue européenne des sciences sociales 124, 336 p.

La société de l'information: la nouvelle frontière? (en coll. avec F. Ischy et O. Simioni). Université de Lausanne, Institut d'anthropologie et de sociologie (coll. «Pratiques et Théories des Sciences et des Techniques» $\mathrm{n}^{\circ}$ VII), $232 \mathrm{p}$.

«La territorialité à l'épreuve de la cyberspatialité». Cahiers géographiques 4: 137-150 (Département de géographie, Université de Genève).

\section{3}

Pour une autre science sociale (éd.). Actes du $\mathrm{XX}^{\mathrm{e}}$ colloque annuel organisé par le Groupe d'Etude «Pratiques Sociales et Théories». Revue européenne des sciences sociales, $127,240 \mathrm{p}$.

«Pour une autre science sociale». Revue européenne des sciences sociales 127 : 5-15.

«A la recherche de l'homme nouveau». Dans L'avenir. Attentes, projets, (dés)illusions, ouvertures, S. Cavalli, J.-P. Fragnière (éds.), Lausanne: Réalités sociales, p. 11-26. 


\section{4}

«Penser l'universalité du don. A quelles conditions?». Revue semestrielle $d u$ M.A.U.S.S. (Paris) 23: 353-376.

«Conformisme de la pensée et exigence d'inventivité». Dans L'invention dans les sciences humaines. Hommage à Giovanni Busino, P. Bridel (éd.), Genève: Labor et Fides, p. 46-65.

\section{5}

«Entre faits et valeurs: portée et limites du colloque». Dans La société de l'information. Récits et réalités (XX $\mathrm{XX}^{\mathrm{e}}$ colloque de l'Académie suisse des sciences humaines et sociales), G. Berthoud, A. Kündig et B. Sitter-Liver (éds), Fribourg: Academic Press, p. XVII-XXII.

«Pervasive Computing: Leading to Posthumanism?». Dans La société de l' information. Récits et réalités, p. 373-386.

«Instrumentaliser le monde pour libérer l'être humain?». Dans La société de l' information. Récits et réalités, p. 455-468.

\section{6}

«Un univers 'kafkaïen' aujourd'hui?». Revue européenne des sciences sociales 133: 13-25.

«Les savoirs aujourd'hui: défis et enjeux». Dans Les sociologues et la philosophie, M. Bassand et Ch. Lalive d'Epinay (éds), Fribourg: Academic Press (sous presse).

«Une philosophie de la concurrence». Catholica (Paris) 91: 13-96.

«Das Universum der Gabe. Anerkennung, Selbstwertschätzung und Dankbarkeit». Dans Geschenkt- Umsonst gegeben? Gabe und Tausch in Ethik, Gesellschaft und Religion, M. Rosenberger (Hrsg.), Bern: Lang (à paraître).

«The Techno-Utopia of 'Human Performance Enhancement'». Dans Utopie Heute. Begriff, Funktion, Bedeutung (XXIII colloque de l'Académie suisse des sciences humaines et sociales), B. Sitter-Liver (Hrsg.), Fribourg: Academic Press (à paraître). 